\title{
Psicología y educación en Chile: problemas, perspectivas y vías de investigación $(1860-1930)^{*}$
}

\section{Psychology and Education in Chile: Problems, Perspectives, and Lines of Investigation (1860-1930)}

\author{
Gonzalo Salas ${ }^{\text {a }}$ \\ Universidad Católica del Maule, Chile \\ ORCID: http://orcid.org/0000-0003-0707-8188 \\ HERNÁN SCHOLTEN \\ Universidad de Buenos Aires, Argentina \\ ORCID: http://orcid.org/0000-0003-3366-2142 \\ Yeniè Norambuena \\ Universidad de Concepción, Chile \\ ORCID: http://orcid.org/0000-0002-4960-2841 \\ Rodolfo Mardones \\ Universidad Austral de Chile, Chile \\ ORCID: http://orcid.org/0000-0003-4027-1027 \\ Ivelisse Torres-Fernández \\ New Mexico State University, Estados Unidos \\ ORCID: http://orcid.org/0000-0002-4573-7597
}

a Autor de correspondencia. Correo electrónico:

gsalas@ucm.cl

Para citar este artículo: Salas, G., Scholten, H., Norambuena, Y., Mardones, R., \& Torres-Fernández, I. (2018). Psicología y educación en Chile: problemas, perspectivas y vías de investigación (1860-1930). Universitas Psychologica, 17(5), 1-14. https://doi.org/ 10.11144/Javeriana.upsy17-5.pecp

\section{RESUMEN}

El presente artículo discute las relaciones entre psicología y educación en Chile entre los años 1860 y 1930. En primer lugar, se abordan las influencias republicanas en la educación y el interés creciente en los fenómenos mentales; posteriormente, se considera el fortalecimiento de la psicología y su rol en los procesos educativos, cuya contribución se constata en los laboratorios de experimentación y la inclusión de la psicología en la formación de profesores. Seguidamente, se discuten aspectos relevantes en la educación de las comunidades indígenas y de las mujeres, para finalizar la exposición con el interés en la psicometría y la aparición de la Escuela Nueva. Todo lo cual favorece el aporte científico de la psicología en la comprensión del educando, que de hecho ha propiciado mejores desarrollos para la sociedad.

\section{Palabras clave}

psicología y educación; historia; enseñanza del indígena; educación de la mujer; psicometría; escuela nueva.

\section{ABSTRACT}

This article discusses the relationships between psychology and education in Chile between the 1860s and 1930s. First, the republican influences on education and the growing interest in mental phenomena are addressed, then the strengthening of psychology and its role in the educational processes, which contributions were noted in research laboratories and the inclusion of psychology in the formation of professors. Subsequently, 
relevant aspects are discussed in the education of indigenous populations and women, to conclude with the interest in psychometrics and the emergence of the New School, all of which allows psychology to better assist in the student's understanding of a scientific context that led to better developments for society.

Keywords

psychology and education; history; indigenous education; women's education; psychometrics; new school.

El presente artículo aborda desde un enfoque histórico la relación entre psicología y educación en Chile. En este sentido, se plantea una caracterización que intenta reconocer, a través de fuentes diversas (libros, artículos, publicaciones periódicas, eventos científicos, etc.), los problemas, perspectivas y vías de investigación que se desarrollaron durante el período que se inicia hacia 1860 y culmina en 1930. Más precisamente, se trata de ilustrar las particularidades de una etapa histórica durante la cual el paradigma positivista y su posterior tránsito hacia la escuela nueva desarrollaron una agenda que favoreció extensas conexiones entre ambas disciplinas y prácticas. Esta mutua vinculación tiene un desarrollo previo, un horizonte que se remonta al menos a la llegada de los dominicos a Chile en 1552 y al posterior arribo de los jesuitas en 1593, quienes, a partir del programa de estudio Ratio Studiorum 1 , impregnaron los temas de filosofía moral, involucrando lecturas de Aristóteles, Kant y Wolff (Hanisch, 1963).

En 1608, el Obispo Fray Juan Pérez de Espinosa fundó el Seminario Conciliar, denominado oficialmente Colegio del Santo Ángel de la Guarda, escuela que mantenían los padres de Santo Domingo y en la que se dictaban cursos elementales de Gramática y Teología. Sin embargo, el primer establecimiento que en el Reino de Chile se propuso instruir sobre los principios de las ciencias no fue aquel, sino el Convictorio de San Francisco Javier (Frontaura y Arana, 1889) que en 1639 comenzó a impartir estudios superiores. Posteriormente, pasó a denominarse Convictorio Carolino y fue colocado bajo la dependencia directa de la
Universidad de San Felipe ${ }^{2}$, fundada en el año 1747 (Medina, 1928).

Con posterioridad, en 1756 y con el impulso de los primitivos franciscanos en Chile, se fundó el Colegio de Chillán ${ }^{3}$, cuyo objetivo fue "propagar las luces de la civilización cristiana entre los salvajes que la habitaban" (Lagos, 1908, p. 1), ya que a su juicio la mayoría de ellos solo estaban entregados a vicios y desórdenes: se puede ver con claridad el espíritu de la época. En este marco, la educación aparece como una eficiente práctica cultural de asimilación, lo cual fue, en primera instancia, extensamente desarrollado por jesuitas, franciscanos y capuchinos con objetivos estratégicos de colonización, basada en la evangelización (Pinto, 1993).

\section{Influencias republicanas en la educación y el interés por los fenómenos mentales}

En pleno desarrollo de la República, los principales educacionistas que se destacaron en el período inicial de la acción estatal, fueron Domingo Faustino Sarmiento y Miguel Luis Amunategui. A partir de 1848, Sarmiento enviaba al ministro Montt un informe de su viaje a Europa y Estados Unidos, durante el cual se ocupó de estudiar la enseñanza de las escuelas normales y primarias (Egaña, 2000). Todos estos aspectos fueron difundidos en El monitor de las escuelas (Sarmiento, 1853), periódico que registraba todo lo referente a la educación primaria, con la finalidad de responder a problemáticas fundamentales a partir del conocimiento de cuántas escuelas y cuántos niños presentes en ellas en relación con el total de población infantil de la República, qué tipo de escuelas existían, cuál la enseñanza impartida en ellas y a cuánto ascendía el gasto de su educación, entre otras premisas. Durante esta etapa, se analizaron los aspectos básicos que permitieron un mayor desarrollo intelectual, y se planteó la necesidad de realizar grandes esfuerzos para poder atender a las condiciones desfavorables que había generado la colonización española (Sarmiento, 1849). 
El fuerte impacto de la corriente positivista, tanto en la psicología como en la educación, se hace claramente apreciable a partir de la década de $1870^{4}$. Basta observar los contenidos de los planes y programas en las escuelas y liceos del país para verificarlo (Celis, Caiceo, López, \& Sánchez, 1982). Esta influencia se debió, en gran medida, a la notable labor previa realizada por los hermanos Juan Enrique y Luis Lagarrigue, quienes trabajaron diversos textos relacionados con esta corriente y expusieron la importancia de adoptar el positivismo como un paradigma de sociedad, basado en las premisas de Auguste Comte, lo cual se refleja en diversas publicaciones (Lagarrigue, J. E., 1884, 1905; Lagarrigue, L., 1894a, 1894b, 1895, 1925, 1946). Estas temáticas impactaron de lleno en los círculos intelectuales chilenos y, especialmente, en las ideas de dos autores capitales: Juan Serapio Lois (pionero de la psicología científica en Chile) y Valentín Letelier (exrector de la Universidad de Chile, uno de los artífices del nuevo modelo de instrucción pública e impulsor de la investigación en psicología), quienes ajustaron estas doctrinas siguiendo un enfoque estrictamente científico. El primero de ellos creó el periódico El Positivista. Periódico Filosófico, Literario, Científico y Moral (Sociedad Escuela Augusto Comte, 1886), que adoptaba las premisas positivistas sobre la disolución de la teología y la metafísica, que, a su juicio, eran totalmente falsas y por tanto estaban condenadas a perderse en el abismo del pasado, dada la nula exactitud y verificación de sus dogmas. Por otra parte, se defendía que la conducta de los individuos debía estar orientada al más pleno desarrollo de sus potenciales, objetivo que figura nuevamente vinculado a la escuela, como el lugar desde donde comenzar a cimentar la moral.

En 1881, se publicó La filosofía del entendimiento, obra póstuma de Andrés Bello, texto que estudia tópicos de psicología mental y lógica, siguiendo los lineamientos de la escuela escocesa y tomando como base el sentido común y la observación para formular un estrecho empirismo (Bello, 1881/1948). A propósito del vínculo advertido entre la disciplina y la constitución de la sociedad (Foucault, 1984), es preciso mencionar la adhesión de Bello al modelo panóptico y la cercanía con Bentham, su impulsor. Bello interviene en el debate público de la época, para promover la instalación de este modelo como método penitenciario y de disciplinamiento del delincuente, en las primeras décadas de la nación independiente (Aedo, 2012) y advierte sobre la utilidad del modelo panóptico aplicado a otros espacios de la sociedad, entre ellos, las escuelas y hospicios (León, 1996), cuestión relevante para interrogar las influencias del modelo carcelario en la escuela chilena, así como el poder civilizador atribuido a las escuelas en la formación de la moral y en la prevención de la conducta delictual.

En 1883, se publica una interesante monografía bajo el título La organización de las escuelas normales (Núñez, 1883), resultado de un informe solicitado por el gobierno chileno al autor, comisionado para estudiar en Europa y América el estado de la instrucción primaria. En dicho documento, se abordan la enseñanza moral, la disciplina escolar, la pedagogía práctica y teórica, e incluso, un análisis sobre la educación de ciegos y sordomudos. Sobre este último tema, el autor dedica un capítulo completo, caracterizando a quienes tenían estas dificultades como "desgraciados", carentes de los medios más indispensables de comunicación con sus semejantes. Si bien Núñez no era muy esperanzador, Chile estaba adelantado en Latinoamérica en estas materias ya que entre 1852 y 1854 se crearon dos escuelas de sordomudos donde se enseñaba a leer, escribir, y se impartían clases de moral religiosa, principios de gramática, costura, bordados y otros trabajos manuales (Caiceo, 2010).

En 1880, tuvo lugar el Congreso Internacional de Enseñanza (Delgado, 1994) en Bruselas, con motivo de la celebración de los cincuenta años de la independencia de Bélgica. En dicho congreso (al cual Núñez asistió en calidad de delegado de Chile), se pusieron en consideración cuestiones concernientes a las escuelas normales y la enseñanza de la mujer (Núñez, 1883). En este lustro, también adquirieron fuerza tópicos concernientes a la higiene escolar, que analizaban las condiciones de la sala de clase (espaciado, iluminación, conservación, aseo, 
etc.), los sistemas de enseñanza, las condiciones individuales y la higiene personal de los alumnos (Dávila, 1884).

Valentín Letelier, por su parte, redactó un segundo informe (posterior al de Núñez) sobre las Escuelas de Berlín, dirigido al "Supremo Gobierno" (Letelier, 1885), con la finalidad de exponer sus observaciones sobre la escuela primaria en temáticas referentes a la administración escolar, la formación de profesores y los métodos didácticos en la enseñanza. Allí se planteaba que en esta época la escuela alemana se dividía entre dos grandes perspectivas metodológicas. La primera, de los ortodojos, compuesta por los descendientes de los teólogos del siglo XVI que fundaron las bases de la instrucción primaria y que tenía como representante principal al Ministro Raumer. Por su parte, la segunda escuela, de los pestalozzistas, contaba entre sus filas con los principales pedagogos de Suiza y Alemania (Pestalozzi, Herbart, Froebel, Diesterweg, Born) y planteaba un cambio de paradigma que apuntaba a lograr un servicio de instrucción pública para la atención de las necesidades del niño, buscando comprender su disposición intelectual y enseñándole solo lo que pudiera comprender naturalmente. El ministro Falk, activo participante en esta polémica, encabezó esta lucha, para así modificar los reglamentos anteriores de Raumer. Dicho informe fue una guía detallada sobre los más relevantes métodos de enseñanza alemanes, que sirvieron como pauta para la reforma pedagógica chilena (Mann, 1910).

A partir de 1886, comienza a difundirse la Jimnasia escolar y se impulsa con fuerza su obligatoriedad, siguiendo la decisión francesa promulgada para la escuela primaria en París en 1880 (Aguirre, 1886), cuya tesis "la gimnasia es al cuerpo, lo que el estudio a la inteligencia" incentivó el desarrollo de las facultades tanto físicas como morales, cuestión que aseguró la preparación de las mentes y los cuerpos para el trabajo y la defensa de la nación.

Ya a finales del siglo XIX se encuentra muy documentado el proceso que culmina (o empieza) con la creación del Instituto Pedagógico de la Universidad de Chile en 1889, durante el gobierno de Balmaceda, por iniciativa de Valentín Letelier. Esta institución fue pionera en la formación de profesores en Latinoamérica y fue allí donde se formaron los grandes maestros que, con posterioridad, desarrollaron su trabajo en las escuelas de todo el país. Para llevar adelante esta iniciativa, Chile se inspiró considerablemente en el caso alemán, como lo demuestran los informes anteriormente citados (Letelier, 1885; Núñez, 1883). La formación del instituto y su condición de "único en su tipo en esta porción del mundo" (Labarca, 1939, p. 251) presentó tal excelencia que, a principios del siglo XX, atrajo a estudiantes de todo el centro sur del continente.

Hacia 1900, el debate en torno a la implementación de tareas escolares fuera del horario escolar (para alumnos de enseñanza secundaria), había llegado al Consejo de Instrucción Pública, organismo que finalmente indicó un tiempo aproximado de ocho horas de trabajo diarias para los educandos, ya que la recarga horaria estaba vinculada con enfermedades corporales y mentales. Adicionalmente, el problema de la fatiga mental se asociaba con la extensión del trabajo intelectual y se criticaba el valor pedagógico atribuido a las tareas y lo innecesario de la carga horaria, especialmente para los más pequeños (Holley, 1900). De cualquier forma, se precisaba que tampoco se podía atribuir al recargo escolar un valor totalitario en los efectos físicos y mentales señalados. En este sentido, el método experimental aparecía como una fuente valiosa de datos y ofrecía elementos de juicio que debían ser considerados para la formulación de políticas públicas.

Una importante publicación periódica se publica por primera vez en 1904. Se trata del mensuario pedagógico La Educación Nacional (Escuela Normal de Preceptores de Santiago, 1904), dirigido por los profesores de la Escuela de Preceptores, que estuvo destinado a servir de portavoz en las escuelas normales. En su primer número, se plantea que el contenido y la alegría que se experimentan en el seno de la escuela es el antídoto contra repugnantes espectáculos que se ofrecen en otros parajes (Retamal, 1904), 
lo cual se vincula con las problemáticas sociales y la posibilidad de redimir a través de la educación los padecimientos que afectaban al pueblo, al mismo tiempo que difundir parámetros del proyecto civilizatorio chileno desde temprana edad (Mardones, Fierro, \& Salas, 2016).

\section{El fortalecimiento de la psicología como ciencia de los procesos educativos}

La psicología aparece nuevamente, aunque con fuerza institucionalizadora en la primera década del siglo XX, con la creación de los laboratorios de psicología en la Escuela Normal de Copiapó (1905) y en el Instituto Pedagógico de la Universidad de Chile (1908). Con mediciones realizadas a escala wundtiana, se intentaba contribuir tanto a la indagación sobre temáticas relacionadas con atención, percepción e inteligencia, para así lograr una modalidad científica de calificación del alumnado que tuviera como fin orientar eficazmente los aprendizajes (Salas, 2012; Salas \& Lizama, 2013). Estas iniciativas fueron lideradas por Rómulo Peña en Copiapó y por el alemán Wilhelm Mann en Santiago.

El conocimiento acuñado sobre la psicología como ciencia y método se encuentra integrado como una parte relevante de la instrucción secundaria en esta década, tal y como muestra El Plan de Estudios $i$ Programas de Instrucción secundaria (Consejo de Instrucción Pública, 1908), que en su programa para la enseñanza de la Filosofía (correspondiente a quinto año de Humanidades) presenta los siguientes contenidos: sensación y percepción; educación de los sentidos, imágenes y representaciones; memoria (desarrollo, importancia y enfermedades); imaginación y atención (factores, cultivo e influjo de otros procesos). Dicho programa tiene continuidad en un segundo ciclo (correspondiente a sexto año de Humanidades), destacando las clasificaciones del conocimiento científico de Auguste Comte y Wilhelm Wundt, así como una sección dedicada al objeto de la psicología y sus métodos: introspección, método objetivo y método experimental. Resulta llamativo que en un apartado especial destinado a la psicología, el plan de estudios incluya contenidos sobre los sentimientos, la vida intelectual y afectiva, el placer y el dolor, volición, libertad, los instintos y sus leyes, hábitos y carácter. En conjunto, el plan de estudios secundarios evidencia un robusto conocimiento de la literatura psicológica y los principales procesos analizados por la disciplina, dando cuenta del lugar concedido a estas materias en la enseñanza.

Por su parte, el Congreso Nacional de Enseñanza Secundaria realizado en 1912, ofrece un segundo tipo de evidencia sobre el importante rol que había adquirido la psicología (como ciencia de las leyes fundamentales de los procesos psíquicos) en el ámbito de la educación. Durante el Congreso, Wilhelm Mann expone su programa de enseñanza de la Filosofía en el que la "psicología tendría un rol central al proporcionar al educando, los medios para reconocer su estilo de trabajo mental y dirigir su personalidad y sus procesos autoeducativos" (Mann, 1912, pp. 26-27). Se entiende entonces, la inclusión de las secciones de psicología en el plan de estudios secundarios y su especial énfasis en el desarrollo y cultivo de los procesos mentales. Bajo este sistema de "enseñanza psicológica escolar", el educando sería capaz de conocer los "factores psíquicos" implicados en el comportamiento y desarrollo, que le ofrece la oportunidad para potenciar sus "aptitudes naturales" y pulir sus debilidades. La enseñanza psicológica enfatiza especialmente el tratamiento de funciones como la memoria, las representaciones, la imaginación, la atención, las funciones lógicas, los sentimientos y la voluntad. Para alcanzar su máxima eficacia, el sistema requería la aplicación de procedimientos de observación de las diferencias individuales, así como de los estados internos mediante la "introspección" y el experimento, contexto en el que Wilhelm Mann propuso la implementación de un gabinete psicológico escolar en las escuelas normales que fuera una extensión e imitara los pasos del ya citado laboratorio de psicología de 1908 (Dirección General de Educación Primaria, 1912). A este respecto, el método no estaba 
centrado únicamente en el perfeccionamiento del individuo, y resulta de sumo interés notar que el sistema y método propuesto poseía un carácter marcadamente social: en primer lugar, proporcionaba una guía a los futuros padres en su rol de educadores y, por otra parte, propiciaba en los educandos el conocimiento de las leyes que gobiernan la cooperación entre los miembros de una comunidad. No obstante, Mann no fue el único en señalar la importancia de los factores psicológicos en la educación, ya que durante el Congreso Nacional de Enseñanza Secundaria de 1912, Luis Galdames presentó varios trabajos que fueron publicados posteriormente en Temas pedagógicos, libro que resume su visión acerca de la necesidad de realizar una reforma nacional de los liceos que considere las diferencias psicológicas de los educandos, junto a las características "raciales" y el entorno cultural (Galdames, 1913) ${ }^{5}$. Al igual que Mann, el autor otorga a la educación un rol central como motor social, aunque, a diferencia de este, discute el impedimento que constituían aquellos factores preexistentes de carácter hereditario, asociados a anormalidades psíquicas o fisiológicas, para obtener el desarrollo del educando.

La importancia de un conocimiento acabado de las patologías de carácter mental y su prevención queda de manifiesto en las páginas de la revista Juventud, creada por la Federación de Estudiantes de la Universidad de Chile que, luego de editarse brevemente entre 1911-1912 y 1918-1921, fue reemplazada por Claridad a partir del año $1920^{6}$. En su primer número, José Ingenieros (1911) publica un artículo que, bajo el título "Clasificación de los delincuentes según su psicopatología", reivindica el concepto unitario de los fenómenos psíquicos y la personalidad humana, para dar cuenta de una concepción psicológica de la conducta criminal. Según la propuesta de este autor, el origen de las conductas inadaptadas se atribuye, principalmente, a deficiencias o trastornos de carácter intelectual (la tríada: moralidad, intelecto y volición). $\mathrm{Si}$ bien Ingenieros reconoce que, en algunos casos, esta inadaptación puede ser de carácter congénito, se inclina más bien a otorgar un papel fundamental a las condiciones de vida en las grandes ciudades que agudizan el problema de la inadaptación de los sujetos. Por este motivo, aboga por la necesidad de promulgar "leyes sociales" (como las que reclamaban las clases obreras) y, principalmente, por la "educación social del niño" 7 . En este sentido, como lo expondrá con más detalles en un texto ulterior, es preciso no solo disponer de una red de instituciones acordes a esta función, sino también "una mejor adaptación de la función escolar a los intereses y necesidades individuales de los niños" (Ingenieros, 1913, p. 248). De este tipo de planteamientos, se desprende la importancia preventiva que comenzaba a otorgarse a la educación y a una adecuada higiene mental, perspectiva que será evidente en publicaciones posteriores.

\section{El "otro" en la educación: la enseñanza del "indígena" y de la mujer}

Una problemática también relevante, tanto para la educación como para la psicología, la constituyen los proyectos de asimilación de los "indígenas chilenos" por medio de la educación. El tema es especialmente relevante si se considera que la "cuestión indígena" era representada en el imaginario político y social como una amenaza a la soberanía de la reciente nación chilena. Para el caso de los mapuches, Tomás Guevara, director del Liceo de Temuco, pedagogo y conocedor de la realidad mapuche en las zonas rurales, declaraba como propósito una contribución al proyecto de convivencia nacional impulsado por el Estado, con el estudio de la raza araucana y la instrucción de los niños y niñas de dichas comunidades, quienes eran considerados como hijos de una "raza inferior" a la raza chilena en cuanto a inteligencia, sentimientos y pasiones (Guevara, 1908). En este contexto, declara procedimientos específicos para la educación de los jóvenes indígenas en los liceos y lamenta sus resultados negativos a propósito de la condición humana. La articulación del positivismo y el paradigma civilización-barbarie dejan en evidencia el proyecto civilizatorio chileno que se instala 
en la Araucanía por medio de la violencia y la masacre por la vía militar (Mardones, 2017), pero también (como queda patente en el caso de Guevara) por la vía de la educación.

Guevara (1908) propone un plan de enseñanza araucana y expone, además, la necesidad de crear escuelas indígenas, en las que se use el idioma castellano por sobre el mapudungún, justificado a partir de sus observaciones, en las que aseguraba constatar una falta de acomodo de los niños indígenas con los "niños nacionales". La creación de establecimientos de enseñanza araucana debían ser instancias graduales: escuelas comunales (dirigidas por un mapuche), regionales (dirigidas por un chileno con ayudante mapuche) y superiores profesionales (dirigidas por un chileno), lo cual aseguraría una progresiva asimilación del indígena por medio de la educación. Para Guevara, era importante que las escuelas fueran internados, lo cual se resume en la siguiente frase: "Las escuelas de los padres capuchinos alemanes $\mathrm{i}$ las de los misioneros evanjélicos tienen alumnos, sobre todo los primeros, por ser internados, i además porque los padres les proporcionan ropa i todo lo que necesitan" (p. 404). Finalmente, expone con certeza que la educación no es suficiente para la "incorporación del mapuche a la vida civilizada" (p. 405), por lo que se requiere que la ley se especialice en el establecimiento de instituciones que aseguren la prosperidad económica. En este punto, se observa que la educación tiene un claro objetivo político sobre territorios recientemente incorporados al Estado de Chile.

Así como se dio la discusión del tema educativo en tierras mapuches, también desde 1868 existieron intentos de crear una escuela de instrucción primaria en Isla de Pascua, esto con anterioridad a 1888 cuando Chile anexiona la isla a su territorio. Sin embargo, y a pesar que desde 1860 existe la Ley de Instrucción Primaria, mediante la cual el Estado se comprometía a proveer escuelas elementales en todo el territorio, no es sino hasta 1914 cuando la escuela y la escolarización occidental llegan a la isla, aunque solo con posteridad aparezcan disposiciones legales del Gobierno con la Ley 3320 (Corvalán,
2015). Tres años después, en 1917, se escribió el libro bilingüe (rapanui-español) El niño pascuense o Te Poki Rapanui, escrito para servir de base a los aborígenes de Isla de Pascua (Vives, 1923).

Es preciso considerar aquí la educación de la mujer, otro campo de discusión y poder que reflejaba antiguas desigualdades. Desde mediados del siglo XIX, se la consideraba desde un ideario que la posicionaba como el núcleo de la familia y donde su condición de madre era el único rol social que legitimaba su aporte al desarrollo del país. Por estos motivos, son escasos los trabajos de educadoras en la primera mitad del siglo XIX, ya que solamente existen menciones al trabajo de Mercedes Cervelló, quien fue recomendada por Andrés Bello como una de las mejores educacionistas de la época (Martínez, 1911), lo mismo sobre Eduvijis Casanova de Polanco, quien escribió sobre educación de la mujer (Casanova de Polanco, 1871, 1876) y fue Directora de la Escuela Superior de Valparaíso. Su trabajo (adoptado por el Gobierno como texto de lectura en las escuelas primarias de niñas) abogó por una instrucción igual a la de los hombres, salvo en las cuestiones referentes a la virilidad, fuerza y representación individual del otro sexo.

Esta situación resulta patente en la restricción de los planes de estudio femenino, en los cursos exclusivos (como costura y labores de mano) así como en la distribución de la carga horaria según género. Sirven de ejemplo las 28 horas de matemáticas que se establecían para los varones contra las 15 que debían recibir las mujeres, situación que se mantuvo hasta 1912 (De Álvarez, 1922). Aunque a fines del siglo XIX (1877) se había logrado el acceso femenino a la instrucción universitaria, una gran cantidad de temas quedaban pendientes, entre ellos, la reforma a los planes de estudios y el problema de la inclusión social.

Como quedó claramente expresado en el Congreso Nacional de Enseñanza Secundaria de 1912, a propósito de la discusión sobre un Reglamento General para los Liceos de Niñas 8 , resultaba imperativo introducir reformas en los planes de estudios femeninos. Amalia de Álvarez, visitadora y directora de 
establecimientos femeninos, realiza importantes aportes a esta discusión, al afirmar que solamente una base educativa homogénea entre hombres y mujeres sería capaz de preparar a estas últimas para desempeñar su rol en la sociedad como ciudadanas ilustradas, necesidad que, poco tiempo más tarde, sería señalada por otra visitadora, Guillermina Von Kalchberg (Von Kalchberg, 1915).

En 1922, se publica Enseñanza secundaria de la mujer (De Álvarez, 1922), documento donde se desarrollan estas ideas y se enfatiza la importancia de la inclusión social en la educación femenina. En este contexto, los liceos debían poseer un carácter "abierto a todas las condiciones sociales" y, además, proveer a las niñas y mujeres un acceso a la escuela y a los planes de estudio que fuesen similares a los recibidos por los varones. De este modo, se expresaba la necesidad de homogeneizar la base educativa de hombres y mujeres. Resulta interesante el énfasis que se otorga a la consideración de los planes de estudios desde una perspectiva más amplia y abarcativa (De Álvarez, 1922), interpelando a un modelo de educación diseñado para hombres, cuestión interesante para evidenciar tensiones de orden social (en este caso la desigualdad de género) en el espacio educativo, tema que también fue desarrollado ampliamente por la connotada educadora Amanda Labarca (Salas, Mardones, Gallegos, \& Ponce, 2014). La continuidad de los estudios a la que se aspiraba con el enlace de la instrucción primaria, secundaria y superior, era considerado un aspecto psicológico importante del sistema de enseñanza, en tanto contribuiría a un aprendizaje continuo y una mayor integración en los procesos de pensamiento (De Álvarez, 1922). Estos planteamientos permiten visualizar una crítica dirigida a un sistema de tipo discontinuo, cuya existencia generaba no solo vacíos de conocimientos, sino también procesos desorganizados de pensamiento, dificultando de este modo un desarrollo armónico de las facultades psicológicas.

En 1920, veinte años después de la redacción del primer proyecto, se publica la Ley de Instrucción Primaria Obligatoria, en la que se establece que, en adelante, la educación primaria debe ser gratuita y obligatoria para ambos sexos (Dirección Jeneral de Educación Primaria, 1921). De esta manera, se amplía la cobertura del sistema, lo que había sido una preocupación para muchos educacionistas por el acceso limitado, tanto de las niñas como de los sectores más empobrecidos.

\section{Del interés psicométrico a la Escuela Nueva}

Los inicios del interés educativo por la psicometría pueden situarse en 1928, con la publicación El desarrollo de la inteligencia medido por el Método Binet-Simon (Tirapegui, 1925). Resulta interesante notar que Tirapegui había introducido este test en el país tres años antes (1925), el que luego fue traducido, adaptado y distribuido en las escuelas de Chile. Como señala el autor, el test fue probado en el Laboratorio de Psicología Experimental durante un año. El libro, editado por el Departamento de Educación Primaria, tuvo por objetivo principal ofrecer a los maestros una técnica científica que permitiera un profundo conocimiento fisiopsicológico de los niños, otorgando así mayor eficiencia a su trabajo.

Este interés tuvo eco en la Revista de Educación, editada por el Ministerio de Educación Pública de Chile. En su primer número, se publica Los efectos de los conflictos mentales sobre la conducta de los niños (Guéritte, 1928), que transcribe la preocupación por la criminalidad infantil y la importancia de la detección temprana de trastornos mentales. Estos trastornos, como critica el autor, podrían encontrar su origen en la crueldad de los sistemas de educación implantados en algunas escuelas, con lo que resulta vital una instrucción que permita introducir los principios de la higiene mental en los establecimientos.

Cambios profundos en materias de psicología y educación se posibilitaron en 1929 con la creación de la Escuela Especial de Desarrollo (Salas \& Inzunza, 2013), la cual señaló el comienzo de la cimentación científica de la escuela primaria en Chile y la educación especial 9 , la cual fue organizada por el profesor Lloyd 
N. Jepsen, perteneciente a la Institución de Vineland. El primer curso de "niños retrasados" se inició en julio de 1929, según la ley que se dio a esta escuela la tarea específica de la atención y educación de los "niños deficientes psíquicos" (Foradori, 1954).

Este tipo de inquietudes resultaba coherente con la publicación de "Enseñanza individualizada” (Salas Marchán, 1929), artículo en el que se reconoce la importancia de la psicología en el conocimiento de las diferencias mentales individuales y, en consecuencia, de los diferentes aspectos de interés que convergen en la conducta de los niños. De estas diferencias, se concluye que el primer deber del profesor es conocer las disposiciones psicológicas de sus educandos y disponer de diferentes métodos de enseñanza, además de diferentes tiempos y ejercicios durante el aprendizaje de los contenidos, desterrando de este modo el método simultáneo. En su aspecto más social, este tipo de propuestas se acompañan del ideal colaborativo en la figura de diferentes formas de actividades y proyectos colectivos o "actividades sociales", quedando en evidencia el valor que se otorga a la "educación social".

La idea de una escuela nueva, preocupada por la educación social y las diferencias individuales de sus educandos, persiste en otros números de la Revista de Educación, lo que puede notarse en el artículo "La disciplina en la Educación" (Paredes, 1929). En él, se recuerda que los niños poseen "fuerzas mentales" diferentes de los adultos y que lo mismo ocurre entre un niño y otro, razón por la que se requiere de un tratamiento pedagógico diferenciado, alejado de la disciplina severa y más cercano a una "disciplina de la libertad". La comprensión del niño como un individuo diferente y activo en su propio aprendizaje, parece haberse establecido en la línea editorial del Ministerio de Educación.

\section{Consideraciones finales}

La relación entre psicología y educación entre los años 1850 y 1930 da cuenta de un proceso de configuración de la educación chilena que transita desde lo disciplinario hacia la comprensión del educando. En las distintas propuestas para el campo educativo, es posible observar implicancias prácticas promovidas desde los avances del conocimiento científico, las que son recogidas por el Estado para establecer adecuaciones en las instituciones educativas. En esta dinámica, la psicología y los saberes psicológicos juegan un rol importante para transitar hacia la comprensión de las capacidades de los niños y adecuar la educación con base en sus características personales. Lo anterior, se puede incluso cotejar y ponderar mediante la publicación de la Bibliografía pedagógica chilena (Ponce, 1902), voluminosa obra, primera de su tipo en Chile, la cual presenta un recuento de temas relacionados con psicología, lógica, ética pedagógica, disciplina escolar, entre otros, cuyos inicios se remontan hasta el año 1829 , aunque con énfasis desde mediados del siglo XIX. De esta forma, se puede vislumbrar panorámicamente un conjunto de propuestas que, desde la psicología y la educación, entrelazando con vínculos la dinámica escolar (Parra, 2015) con los fenómenos psicológicos, posibilitaron vías de investigación dinamizadoras de los debates de la época. Adicionalmente, es posible observar como interactúa el saber psicológico especializado en la normalización requerida como meta estratégica del Estado. Este hecho se acentúa en la asimilación de mapuches en el sur de Chile, donde la educación se posiciona como una estrategia política para expandir el proyecto civilizatorio nacional a territorios colonizados por el Estado de Chile por medio de la violencia.

Cabe mencionar que la educación chilena creció de forma importante desde la creación del Instituto Pedagógico de la Universidad de Chile, ya que su impronta, sello y tradición lograron que los estudiantes que pasaron por sus aulas irradiaran su formación en diversas escuelas de todo del país. El enfoque psicológico del Instituto fue de alto calibre y desarrolló una impronta alemana que dirigió los pasos de la educación chilena para luego volcar la mirada hacia Estados Unidos y posteriormente otorgar una mirada nacionalista que enalteció los ideales de la República y la educación nacional, con 
los aportes y discusiones pedagógicas surgidas desde nuestros propios contextos y actores, lo que se reflejaba en hacer de nuestra patria "unos nuevos Estados Unidos de América, una nueva Alemania, una aún más nueva Inglaterra" (Molina, 1912, p. 12).

Por su parte, las influencias del positivismo en Chile fueron amplias, sobre todo en la implementación del método científico, lo cual se puede expresar a través del auge de la psicología experimental y los laboratorios que permitían medir los procesos psicológicos para así lograr datos verificables y contrastables; sin embargo, este proceso fue en decadencia desde la segunda década del siglo XX. Uno de sus detractores fue Enrique Molina quien, desde 1919, se dedicó a actividades de enseñanza en la Universidad de Concepción y fue un importante divulgador de la obra de Bergson (Kempff, 1958), además el Gobierno le confió entre octubre de 1918 y junio de 1919 una comisión para visitar las principales universidades de Estados Unidos (Molina, 1921), lo cual tuvo impacto a su regreso, dado que buscó siempre conciliar lo científico con lo cultural para así proporcionar alimento a las inteligencias, logrando integrar una mirada filosófica que irradiaba sus cosmovisiones educativas. Por otra parte, el trabajo de Amanda Labarca, (quien también transitó diversas universidades norteamericanas) provocó un giro hacia la obra de William James y John Dewey, quienes presentaron una mirada de la psicología orientada hacia las problemáticas sociales aplicadas (Salas et al., 2014), lo cual trascendió en el campo de la educación nacional, y a partir de la tercera década del siglo XX generó una nueva etapa donde el laboratorio pasó a ser la propia escuela. Ya en la década de los 40, con la emergencia del Instituto de Psicología de la Universidad de Chile en 1941, comienza a desplegarse la investigación aplicada a la diversidad de áreas de la psicología.

Finalmente, se debe mencionar que el presente estudio puede generar una nueva vía hacia estudios historiográficos que podrían profundizar temáticas como la inteligencia, la escuela nueva, la educación de la mujer, educación especial, tratamientos de "anormales", entre otros, todo lo cual permitirá fortalecer el corpus que el presente trabajo releva.

\section{Referencias}

Aedo, M. (2012). Panóptico, novela y sociedad moderna en Chile y América Latina: El pirata del Huayas (1855), de Manuel Bilbao. Atenea, 505, 263-283. https://doi.or $\mathrm{g} / 10.4067 / \mathrm{S} 0718-04622012000100012$

Aguirre, J. J. (1886). Manual de jimnasia escolar. Para el uso de las escuelas de instrucción primaria. Santiago de Chile: Imprenta y Litografía de Pedro Cadot y Compañía.

Alvarado, M. (2016). Revistas culturales y literarias chilenas de 1900 a 1920: Legitimadoras de campo literario nacional. Santiago de Chile: Cuarto Propio.

Barrera, F. (2010). Orígenes de la psicología educacional chilena. En M. A. Laborda \& V. E. Quezada (Eds.), Notas históricas de la psicología en Chile (pp. 163-191). Santiago de Chile: Universitaria.

Bello, A. (1948). Filosofía del entendimiento. México: Fondo de Cultura Económica.

Casanova de Polanco, E. (1871). Educación de la mujer. Valparaíso: Imprenta de la Patria.

Casanova de Polanco, E. (1876). Elementos de economía doméstica e higiene casera. Santiago de Chile: Imprenta de la Librería del Mercurio.

Caiceo, J. (1988a). La educación especial en Chile: un esbozo de su historia. Revista de Pedagogía, 306, 47-50.

Caiceo, J. (1988b). La educacio\#n especial en Chile: periodo de consolidación. Revista de Pedagogía, 309, 135-141.

Caiceo, J. (2010). Esbozo de la educación especial en Chile: 1850-1980. Revista de Educación y Pedagogía. 22(57), 31-49. Recuperado de http://aprendeenlinea.udea.edu.co/revis tas/index.php/revistaeyp/article/view/9839

Castro Boniche, R. (2015). Historia de la educación diferencial en Chile y la región de Coquimbo. La Serena, CL: Universidad de La Serena. 
Celis, L., Caiceo, J., López, S., \& Sánchez, E. (1982). La presencia de la filosofía en la Universidad Católica (1888-1973). Anales de la Escuela de Educación, 5, 1-215.

Consejo de Instrucción Pública. (1908). Plan de Estudios $i$ Programas de Instrucción Secundaria. Santiago de Chile: Imprenta Cervantes.

Corvalán, J. (2015). Educación en Rapa Nui. Sociedad y escolarización en Isla de Pascua. Santiago de Chile: Ediciones Universidad Alberto Hurtado.

Dávila, R. (1884). La hijiene de la escuela. Santiago de Chile: Imprenta Cervantes.

De Álvarez, A. (1922). Enseñanza secundaria de la mujer. Régimen de los liceos de niñas. Rancagua, CL: Imprenta Bellavista.

Delgado, B. (1994). La educación en la España contemporánea. Madrid: Morata.

Dirección General de Educación Primaria. (1912). Revista de Instrucción Primaria, 26, 4. Santiago de Chile: Autor.

Dirección Jeneral de Instrucción Primaria. (1921). Lei N. 3,654 sobre educación primaria obligatoria. D. O. N. ${ }^{\circ} 12,755$ de 26 de agosto de 1920. Santiago de Chile: Imprenta Lagunas \& Co.

Egaña, M. L. (2000). La educación primaria popular en el siglo XIX en Chile. Una práctica de politica estatal. Santiago de Chile: Lom Ediciones.

Escuela Normal de Preceptores de Santiago. (1904). La educación nacional. Mensuario pedagójico. Órgano de la Escuela Normal de Preceptores de Santiago. Santiago de Chile: Imprenta Cervantes.

Foradori, A. (1954). La psicología en América. Buenos Aires: Instituto Cultural Joaquín González.

Foucault, M. (1984). Vigilar y castigar. El nacimiento de la prisión. México: Siglo XXI.

Frontaura y Arana, J. M. (1889). Historia del Convictorio Carolino. Santiago de Chile: Imprenta Nacional.

Galdames, L. (1913). Temas pedagógicos. Santiago de Chile: Imprenta Universitaria.
Guéritte, M. T. J. (1928). Los efectos de los conflictos mentales sobre la conducta de los niños. Revista de Educación, 1(1), 7-9.

Guevara, T. (1908). Psicolojía del Pueblo Araucano. Santiago de Chile: Imprenta Cervantes.

Hanisch, W. (1963). En torno a la filosofía en Chile (1594-1810). Santiago de Chile: Universidad Católica de Chile.

Herrera, V. (2010). Estudio de la población sorda en Chile: evolución histórica y perspectivas lingüísticas, educativas y sociales. Revista Latinoamericana de Educación Inclusiva, 4(1), 212-226. Recuperado de http://www.rinace.net/rlei/ numeros/vol4-num1/art10.pdf

Holley, G. (1900). Sobre el recargo escolar. La revista de Chile, 5(1), 1-2. Recuperado de http://www.memoriachilena.cl/602/w3article-87310.html

Ingenieros, J. (1911). Clasificación de los delincuentes según su psicopatolojía. Juventud, Revista de la Federación de estudiantes de Chile, 1(4), 177-184.

Ingenieros, J. (1913). Criminología. Madrid: Daniel Jorro.

Kempff, M. (1958). Historia de la filosofía en Latinoamérica. Santiago de Chile: Editorial Zig Zag.

Labarca, A. (1939). Historia de la enseñanza en Chile. Santiago de Chile: Imprenta Universitaria.

Labarca, A. (1947). Feminismo contemporáneo. Santiago de Chile: Editorial Zig Zag.

Labrador, C. (1999). La Ratio Studiorum de 1599. Un sistema educativo singular. Revista de Educación, 399, 117-134. Recuperado de ht tps://www.mecd.gob.es/dctm/revista-de-ed ucacion/articulosre319/re3190607777.pdf? documentId=0901e72b812709b5

Lagarrigue, J. E. (1884). El tránsito a la religión de la humanidad. Santiago de Chile: Imprenta Cervantes.

Lagarrigue, J. E. (1905). Lo sobrenatural ante el positivismo. Año 51 de la Era Normal. Santiago de Chile: Imprenta Cervantes.

Lagarrigue, L. (1894a). 37. ${ }^{\circ}$ aniversario de la muerte de Auguste Comte. Año 106 de la 
Gran Crisis. Santiago de Chile: Editorial Ercilla.

Lagarrigue, L. (1894b). Programa de la Sociedad Positivista de Chile. Año 106 de la Gran Crisis. Santiago de Chile: Editorial Ercilla.

Lagarrigue, L. (1895). Celebración de la humanidad. Año 107 de la Gran Crisis. Santiago de Chile: Editorial Ercilla.

Lagarrigue, L. (1925). La propiedad. Positivismo y comunismo. Santiago de Chile: Imprenta Cervantes.

Lagarrigue, L. (1946). Religión Universal. Síntesis subjetiva o industria. Santiago de Chile: Fundación Juan Enrique Lagarrigue.

Lagos, R. (1908). Historia de la Misiones del Colegio de Chillán (Vol. 1). Barcelona: Herederos de Juan Gili.

León, M. A. (1996). Sistema carcelario en Chile. Visiones, realidades y proyectos (1816-1916). Santiago de Chile: Dirección de Bibliotecas, Archivos y Museos.

Letelier, V. (1885). Las escuelas de Berlín. Santiago de Chile: Imprenta Nacional.

López, A. (1914). Colegio de Chillán. Madrid: Archivo Ibero-Americano.

Mann, W. (1910). La educación alemana en la educación chilena en general. En Los alemanes en Chile. Homenaje de la Sociedad Científica Alemana de Santiago a la Nación Chilena en el Centenario de su Independencia (Vol. 1, pp. 102-154). Santiago de Chile: Imprenta Universitaria.

Mann, W. (1912). La enseñanza de la filosofía en el Liceo. Santiago de Chile: Imprenta Universitaria.

Mardones, R. E. (2017). Crisis moral y discursos sobre la psicología del sujeto mapuche a inicios del siglo XX en Chile. En R. E. Mardones (Ed.), Invención de la psique nativa. Construcción discursiva de las características psicológicas atribuidas al sujeto indígena en América Latina (pp. 201-244). Santiago de Chile: RIL editores/Editorial Universidad Santo Tomás.

Mardones, R. E., Fierro, C., \& Salas, G. (2016). Cuestión social en Chile. Discursos sociales y sus referencias a los saberes "psi” (1880-1930). Revista de Historia de la Psicología, 37, 8-15. Recuperado de https://www.revistahistoriapsicologia.es/ revista/2016-vol-37-n\%C3\%BAm-1/

Martínez, M. E. (1911). Mujeres célebres de Chile. Santiago de Chile: Imprenta Santiago.

Medina, J. T. (1928). Historia de la Real Universidad de San Felipe. Santiago de Chile: Imprenta y Litografía Universo.

Mellafe, R., \& González, M. T. (2007). El Instituto Pedagógico de la Universidad de Chile. Su aporte a la educación, cultura e identidad nacional (1889-1981). Santiago de Chile: Lom Ediciones.

Molina, E. (1912). La cultura i la educación jeneral. Santiago de Chile: Imprenta Universitaria.

Molina, E. (1921). De California a Harvard. Estudio sobre las universidades norteamericanas y algunos problemas nuestros. Santiago de Chile: Sociedad Imprenta y Litografía Universo.

Núñez, A. (1883). La organización de las escuelas normales. Santiago de Chile: Imprenta de la Librería Americana.

Palacios, N. (1904). Raza chilena: libro escrito por un chileno $i$ para los chilenos. Valparaíso: Imprenta y Litografía Alemana.

Paredes, A. (1929). La disciplina en la educación. Revista de Educación, 1(4), 213-217.

Parra, D. (2015). Un análisis historiográfico sobre las relaciones entre psicología y educación en Chile. Revista de Historia de la Psicología, 36(2), 95-114. Recuperado de https://www.revistahistoriapsicologia.es/ revista/2015-vol-36-n\%C3\%BAm-2/

Pinto, J. (1993). Jesuitas, franciscanos y capuchinos italianos en la Araucanía (1600 - 1900). Revista Complutense de Historia de América, 19, 109-147. Recuperado de http://europa.sim.ucm.es/co mpludoc/AA?articuloId $=690934$

Ponce, M. A. (1902). Bibliografía pedagógica chilena. Santiago de Chile: Imprenta Elzeviriana.

Retamal, M. (1904). Los malos ejemplos en la educación moral. La Educación Nacional, 1(1), 7-15.

Salas Contreras, G. (2012). La influencia europea en la historia de la psicología en Chile. 
Interamerican Journal of Psychology, 46(1), 99-110. Recuperado de http://www.redalyc .org/pdf/284/28424858010.pdf

Salas Contreras, G., \& Inzunza, J. (2013). Antecedentes históricos de la psicología educacional en Chile. En C. Cornejo, P. Morales, E. Saavedra \& G. Salas Contreras (Eds.), Aproximaciones en psicología educacional. Diversidades ante la contingencia actual (pp. 27-41). Talca, CL: Universidad Católica de Maule.

Salas Contreras, G., \& Lizama, E. (2013). Historia de la psicología en Chile. 1889-1981 (2.a ed.). La Serena, CL: Universidad de La Serena.

Salas Contreras, G., Mardones, R., Gallegos, M., \& Ponce, F. P. (2014). Amanda Labarca (1886-1975) y sus referencias psicológicas en el contexto educativo en Chile. Universitas Psychologica, 13(5), 2059-2068. https://doi.org/10.11144/Javeri ana.upsy13-5.alrp

Salas Marchán, M. (1929). Enseñanza individualizada. Revista de Educación, 1(2), 82-83.

Sarmiento, D. (1849). De la educación popular. Santiago de Chile: Imprenta de Julio Belin i Compañía.

Sarmiento, D. (1853). El monitor de las escuelas primarias (Vol. 1). Santiago de Chile: Imprenta de Julio Belin i Compañía.

Sociedad Escuela Augusto Comte. (1886). El Positivista. Periódico filosófico, literario, científico y moral (J. S. Lois, Ed.). Copiapó, CL: El Atacameño.

Tirapegui, L. A. (1925). El desarrollo de la inteligencia medido por el método Binet-Simon. Anales de la Universidad de Chile, 453-591. Recuperado de http://www.revistas.uchile.cl/index.php/ ANUC/article/view/25591/26960

Vives, J. I. (1923). Te Poki Rapanui (El niño pascuense). Santiago de Chile: Imprenta Cervantes.

Von Kalchberg, G. (1915). Los Liceos de Niñas. Santiago de Chile: Imprenta y Encuadernación El Globo.

\section{Notas}

1 Para conocer más detalles, se sugiere leer el artículo de Labrador (1999), escrito en conmemoración de los 400 años de la aprobación definitiva del documento Ratio Studiorum, el cual comprende el sistema educativo de la Compañía de Jesús.

2 La creación de la Universidad de San Felipe fue una labor colectiva fundada a imitación de la Universidad de Salamanca y la Universidad Nacional Mayor de San Marcos (Mellafe \& González, 2007).

3 El Colegio se denominó San Ildefonso de la ciudad de San Bartolomé de Chillán, también conocido como Colegio Seminario (López, 1914).

4 En el trabajo Orígenes de la psicología educacional chilena: 1885-1919 (Barrera, 2010) se habla del giro filosófico-positivista, al evidenciarse la separación de la psicología y la filosofía, y cómo estos principios se comienzan a aplicar en el campo de la educación. El autor analiza, en el período positivista, cuatro textos de Wilhelm Mann escritos entre los años 1905 y 1919.

5 Recuérdese la importancia que comenzaba a adquirir la noción de "raza" en Chile durante este periodo, que tiene al libro Raza chilena como uno de los principales en la materia (Palacios, 1904).

6 Para ver más detalles sobre este tipo de publicaciones se sugiere el libro de Marina Alvarado, Revistas culturales y literarias chilenas de 1900 a 1920. Es importante mencionar que existió otra revista denominada La Juventud, fundada en 1901 por Joaquín Edwards Bello y Alberto Díaz, que alcanzó solamente cinco números de publicación. Por su parte, Claridad tuvo una primera etapa de edición entre 1920 y 1926 para, luego de un breve resurgimiento a comienzos de la década 1930, finalizar definitivamente su publicación (Alvarado, 2016).

7 Como lo plantea en su Criminología: "Urge cuidar la planta desde la semilla, sin esperar que haya retoñado siniestramente: hay que prevenir la delincuencia protegiendo á la infancia, haciendo de su salud física y de su adaptación moral la más grave preocupación de la sociedad. Hacia la 
infancia enfermiza, física ó moralmente, deben converger los mayores esfuerzos de una generosa protección social" (Ingenieros, 1913, pp. 246-247).

8 La fundación del Liceo de Niñas n. 1 de Santiago data de 1895, aunque le habían precedido desde 1854 las escuelas normales de mujeres y desde 1888, las escuelas técnicas (Labarca, 1947).

9 Sobre historia de la educación diferencial, se sugiere la lectura del libro Historia de la educación diferencial en Chile y la región de Coquimbo (Castro Boniche, 2015) y los artículos "La educación especial en Chile: un esbozo de su historia" (Caiceo, 1988a), "La educación especial en Chile: periodo de consolidación” (Caiceo, 1988b), "Esbozo de la educación especial en Chile: 1850-1980" (Caiceo, 2010) y específicamente sobre población sorda, "Estudio de la población sorda en Chile: Evolución histórica y perspectivas lingüísticas, educativas y sociales" (Herrera, 2010).

* Artículo de investigación. El presente estudio ha sido financiado con la ayuda para proyectos de investigación concedida por el Fondo Nacional de Desarrollo Científico y Tecnológico del Gobierno de Chile (FONDECYT n.․ 11150436), denominado "Antecedentes, escenarios e itinerarios de la psicología educacional en Chile en el período pre-profesional (1860-1946)", dirigido por el primer autor del artículo. 